\title{
Status of high sensitive C- reactive protein (hs-CRP) in NAFLD obese type-2 diabetic subjects
}

\author{
Sanjeev Kumar Singh¹, Dharmveer Sharma ${ }^{2, *}$, Nivedita Singh³, Raj Kumar ${ }^{4}$, Pavan Gautam ${ }^{5}$ \\ 1,3Professor, Dept. of Biochemistry, G.R. Medical College, Gwalior, Madhya Pradesh, ${ }^{2}$ Associate Professor, Dept. of \\ Biochemistry, Government Medical College, Shivpuri, Madhya Pradesh, ${ }^{4}$ Associate Professor, Dept. of Biochemistry, B.R.D., \\ Medical College Gorakhpur, Uttar Pradesh, ${ }^{5}$ Lecturer, Dept. of Biochemistry, Government Medical College, Banda, Uttar \\ Pradesh, India
}

*Corresponding Author:

Email: dharmveersharma434@gmail.com

Received: $4^{\text {th }}$ February, 2018

Accepted: $3^{\text {rd }}$ April, 2018

\begin{abstract}
Introduction: Non alcoholic fatty liver disease (NAFLD) is a disease occurring in patients without any significant alcohol consumption. It includes a broad spectrum and advanced forms of liver diseases, from simple steatosis to nonalcoholic steatohepatitis, fibrosis, and cirrhosis and is associated with diabetes mellitus, insulin resistance, obesity, dyslipidemia and metabolic syndrome. The aim of this study is to evaluate the metabolic significance of NAFLD in obese and type-2 diabetic subjects compare to healthy group of individuals and those independent factors associated with NAFLD.

Materials and Methods: Our study was conducted in Govt. Medial College, Banda (UP) as well as G. R. Medical College, Gwalior (MP). A total of 200 subjects were involved in our study, diagnosis being based on ultrasonography. Out of these, 100 were obese with type- 2 diabetic patients and 100 were healthy subjects. They were evaluated by measurement of BMI and several biochemical blood parameters such as fasting blood glucose, lipid profile, liver function tests (ALT, ALP and GGT) and hs-CRP level.

Results: An increase in the BMI and levels of FBS, total cholesterol, triglycerides, LDL, VLDL, GGT, ALT, ALP and hs-CRP level and a decrease in HDL was observed in NAFLD group of individuals. The values of these biochemical markers were found higher in NAFLD patients and the variations were found to be statically significant. BMI and triglycerides were positively correlated with fatty liver.

Conclusion: Obesity, hyperglycemia, dyslipidemia and increased liver enzymes and hs-CRP level are seen more commonly in non alcoholic fatty liver in type-2 diabetic obese patients.
\end{abstract}

Keywords: NAFLD, BMI, High sensitive C-reactive protein.

\section{Introduction}

Non alcoholic fatty liver disease (NAFLD) describes a clinicopathological condition that is described by significant lipid deposition in the hepatocytes of the liver parenchyma in patients with no history of excessive intake of alcohol. The spectrum of NAFLD is broad, ranging from a simple steatosis to nonalcoholic steatohepatitis, fibrosis and cirrhosis. Obesity, dyslipidemia, insulin resistance and diabetes mellitus are well known risk factors for the development of a fatty liver disease. ${ }^{1,2}$ NAFLD includes patients with simple steatosis and non-alcoholic steatohepatitis, which can lead to cirrhosis and hepatocellular carcinoma. ${ }^{3}$ Another inflammatory marker, hs-CRP has given mixed results as a biomarker while interleukin-6 (IL-6) has been shown to distinguish differentiate between disease states and independently correlates with fibrosis. ${ }^{4,5}$

\section{Materials and Methods}

Subjects were randomly taken from those attending the medical outpatient department (OPD) of Government Medial College, Banda (UP) as well as G.R. Medical College, Gwalior (MP). A total number of 200 cases were included in our study, diagnosis being based on ultrasonography. Out of these, 100 were non obese and non diabetic healthy controls and 100 were obese with type-2 diabetic patients of both sexes male and female included in our observation, except based on significant alcohol consumption (>20 g/day). A written consent was obtained from the patients. The approval was taken from the institutional ethics committee for conducting the study. Subject was instructed to stand still on the platform, with the body weight evenly distributed between both the feet, for the measurement of weight. Weight (Seca 803, digital scale, Germany) was measured to the nearest of $0.1 \mathrm{~kg}$. Stadiometer (Seca 206, Germany) was used for the measurement of height with head held in Frankfort plane to the nearest of $0.1 \mathrm{~cm}$. BMI was calculated by the formula i.e. weight $(\mathrm{kg}) /$ height $\left(\mathrm{m}^{2}\right)$.

The patients were then evaluated by the measurement of Body Mass Index (BMI), FBS (Fasting Blood Sugar), TC (Total Cholesterol), TG (Triglycerides), LDL (Low Density Lipoprotein), HDL (High Density Lipoprotein), VLDL (Very Low Density Lipoprotein), TB (Total Bilirubin), ALT (Alanine Aminotransferase), GGT (Gamma Glutamyl Transpeptidase), ALP (Alkaline Phosphatase), and hsCRP. The data was analysed by Statistical packages for 
social science (SPSS version 21.0). Mean and standard deviation were analysed for quantitative variables like BMI, FBS, TC, TG, LDL, HDL, VLDL, Total Bilirubin, ALT, GGT, ALP and hs-CRP. To compare mean of all the quantitative variables between the two groups the independent sample t-test of patients were 0.001 level was considered significant. Correlation coefficient analysis was performed for risk factors of NAFLD. P-value less than 0.05 was considered statistically significant.

\section{Results}

In our study, 100 patients of type-2 diabetes mellitus obese subjects and 100 healthy controls were included. The average age of the patients was $52 \pm 8$ years (Ranging from 36 to 74). Comparison of means of serum biochemical markers between fatty liver and healthy groups is presented in Table. The values of all these biochemical parameters except HDL were increased in fatty liver disease patients in comparison to healthy group and the variations were found to be statistically significant $(\mathrm{P}$ value $<0.01$ and $<0.001)$. BMI and triglycerides were positively correlated with nonalcoholic fatty liver disease.

Table: Comparison of laboratory abnormalities between non-alcoholic fatty liver disease and healthy controls

\begin{tabular}{|l|c|c|c|c|}
\hline \multicolumn{1}{|c|}{ Study Variables } & Healthy controls n=100 & NAFLD n=100 & t values & P values \\
\hline BMI & $22.6 \pm 1.88$ & $32.85 \pm 3.89$ & 4.048 & $\mathrm{P}<0.001^{* *}$ \\
\hline FBS & $87.46 \pm 13.46$ & $148.0 \pm 37.7$ & 6.426 & $\mathrm{P}<0.001^{* *}$ \\
\hline $\begin{array}{l}\text { Total Cholesterol } \\
\text { (mg/dl) }\end{array}$ & $186.3 \pm 21.09$ & $248.4 \pm 41.9$ & 4.234 & $\mathrm{P}<0.001^{* *}$ \\
\hline TG (mg/dl) & $128.09 \pm 19.49$ & $212.7 \pm 55.05$ & 8.549 & $\mathrm{P}<0.001^{* *}$ \\
\hline HDL (mg/dl) & $47.45 \pm 6.14$ & $33.27 \pm 7.02$ & 3.267 & $\mathrm{P}<0.001^{* *}$ \\
\hline LDL (mg/dl) & $114.1 \pm 20.20$ & $161.7 \pm 39.3$ & 7.384 & $\mathrm{P}<0.001^{* *}$ \\
\hline VLDL (mg/dl) & $23.58 \pm 4.09$ & $52.89 \pm 11.3$ & 6.725 & $\mathrm{P}<0.001^{* *}$ \\
\hline ALT (IU/L) & $27.34 \pm 6.44$ & $54.16 \pm 16.1$ & 5.362 & $\mathrm{P}<0.001^{* *}$ \\
\hline ALP (IU/L) & $179.7 \pm 336.75$ & $292.6 \pm 21.0$ & 7.180 & $\mathrm{P}<0.001^{* *}$ \\
\hline GGT (IU/L) & $25.05 \pm 5.24$ & $49.39 \pm 9.15$ & 6.752 & $\mathrm{P}<0.001^{* *}$ \\
\hline hs-CRP (mg/L) & $1.03 \pm 0.46$ & $3.66 \pm 1.20$ & 7.902 & $\mathrm{P}<0.001^{* *}$ \\
\hline $\begin{array}{l}\text { Total bilirubin } \\
\text { (mg/dl) }\end{array}$ & $0.72 \pm 0.26$ & $1.22 \pm 0.50$ & 2.567 & $\mathrm{P}<0.01^{*}$ \\
\hline
\end{tabular}

* Significant at $\mathrm{P}<0.01, * *$ significant at $\mathrm{P}<0.001$

\section{Discussion}

The prevalence of NAFLD is highly associated with insulin resistance, type-2 diabetes mellitus, obesity, dyslipidemia and metabolic syndrome. ${ }^{6}$ The presence of NAFLD correlates significantly with BMI. ${ }^{7}$ In our study only BMI was taken as a marker for obesity, raised BMI indicated strong correlation with presence of nonalcoholic fatty liver. In obese type- 2 diabetic fatty liver group, the mean BMI was $32.85 \pm$ 3.89 but in non-fatty liver group it was $22.6 \pm 1.88$ (P value $<0.01$ ). In literature, the prevalence of NAFLD has been found to be $100 \%$, among severe obese patients with type- 2 diabetes mellitus patients. ${ }^{8}$ Visceral obesity is frequently associated with NAFLD and their coexistence in the same individual subject increases the chances of having more advanced forms of liver disease. NAFLD is found in 60\%-95\% of people with obesity. ${ }^{9}$ The correlation between fatty liver, impaired glucose tolerance, diabetes mellitus and hyperlipidemia is well recognized. It has been established that insulin resistance can lead to increased free fatty acid in the liver, subsequently higher triglyceride synthesis and increased secretion of triglyceride rich VLDL from the liver. Hypertriglyceridemia have been also strongly associated with liver fat accumulation., ${ }^{8,10,11}$ Our study showed FBS levels in fatty liver disease group (mean $148.0 \pm 37.7$ ) were higher than healthy control group (mean 87.46 \pm 13.46 ), which confirmed the obvious dysglycemia in these patients ( $\mathrm{P}$ value $<0.001$ ). We also observed that increased triglyceride levels (mean $212.7 \pm 55.05)$ in diabetic fatty liver group in comparison to healthy group (triglycerides mean $128.09 \pm 19.49)$ and the results were statistically significant ( $\mathrm{P}$ value $<0.001)$. In a correlation coefficient analysis triglycerides were also found to be increase in obese type 2 diabetic obese nonalcoholic fatty liver population. The study was done in China also found that fatty liver positively correlated with plasma triglyceride levels and negatively with plasma HDL-C level. ${ }^{12}$ In our study also, the elevated total cholesterol positively correlated with fatty liver disease. We also found that increased levels of ALT, ALP and GGT in obese type 2 diabetic NAFLD subjects in comparison to healthy group and the results were statistically significant ( $\mathrm{P}$ value $<0.001$ ). This is also reported in other studies as well. ${ }^{13-15}$ In this study, though raised ALT levels are taken as the first marker of fatty infiltration of the liver. ${ }^{16}$ The mechanism behind the increased secretion of liver enzymes in NAFLD patients is that dyslipidemia and insulin resistance 
leading to significant lipid deposition in hepatocytes causes initiation of mitochondrial swelling, increased lysosomal fragility and impaired membrane integrity, resulting release of hepatic enzymes from the damaged hepatocytes. ${ }^{8}$ Furthermore, it is concluded that fasting blood glucose level was an independent predictor of nonalcoholic fatty liver disease. ${ }^{17}$ This deviation between our data to that of Marchesini et al implies that the clinicopathological profile of Indian NAFLD patients are different from that seen in other ethnic group of individuals. ${ }^{17,18}$ The present study also, revealed significant higher levels of serum high sensitive C-reactive protein in NAFLD female and male patients (mean 3.66 \pm 1.20 ) in comparison to those of their corresponding healthy controls (mean $1.03 \pm 0.46$ ) with significant higher levels in female patients compared to those levels of male patients. These findings similar with those of previous studies. ${ }^{19-21} \mathrm{hs}-$ CRP levels have been shown to be closely related to obesity, in particular central or visceral fat deposition. $^{22,23}$ The study of Koruk et al. found that increased levels of hs-CRP could be helpful in the diagnostic work-up of patients with fatty liver disease. ${ }^{24}$ Moreover added that hs-CRP could be a clinical feature that not only distinguishes (Non-alcoholic steatohepatitis) NASH from simple non-progressive steatosis but also indicates the severity of hepatic fibrosis. ${ }^{25}$ Bilirubin levels may also be linked to NAFLD via fatty acid metabolism. Higher de novo lipogenesis and peripheral fatty acids mainly derived from lipolysis of adipose tissue contribute to the accumulation of hepatic fat in NAFLD. ${ }^{26,27}$

\section{Conclusion}

The occurrence of NAFLD is high in type-2 diabetic patients, insulin resistance and obesity, dyslipidemia, dysglycemia, increased secretion of liver enzymes (ALT, ALP and GGT) and hs-CRP is seen more frequently in fatty liver than in non-fatty liver subjects. The associated risk factors for diabetic fatty liver are the raised BMI and increased levels of triglycerides.

\section{Limitation}

Our study also came up with few limits. The diagnosis of NAFLD in the study was based on ultrasonography (USG) and exception of the known causes of chronic liver disease, but this was not confirmed by liver biopsy, in our study. Some clinical trials regarding use of other inflammatory markers also prove significant.

\section{Acknowledgement}

The authors are grateful to the Principal and Dean, Head of Department of Medicine who have provided proper prompts and reference services for the study and Government Medical College, Banda (U.P.) and G.R.
Medical College, Gwalior (M.P.) for granting acquiescence and providing facilities for our research.

Conflict of Interest: There has been no conflict of interest at any stage of the study.

\section{References}

1. Greenfield, Cheung O, Sanyal AJ: Recent advances in nonalcoholic fatty liver. Curr Opin Gastroenterol. 24:320, 2008.

2. Kim JH, Kim JH. Metabolic significance of nonalcoholic fatty liver disease in non obese, non diabetic adults. AMA. 2004;164:2169-2175.

3. Sanyal AJ, Banas C, Sargeant C, Luketic VA, Sterling RK, Stravitz RT. Similarities and differences in outcomes of cirrhosis due to non-alcoholic steatohepatitis and hepatitis C. Hepatology. 2006;43:682-689.

4. Tarantino G, Conca P, Pasanisi F, et al. could inflammatory markers help diagnose nonalcoholic steatohepatitis. Eur J Gastroenterol Hepatol. 2009;21:504-11.

5. Lemoine M, Ratziu V, Kim M. Serum adipokine levels predictive of liver injury in non-alcoholic fatty liver disease. Liver Int. 2009;29:1431-8.

6. Angulo P. Non alcoholic fatty liver disease. $N$ Eng J Med. 2002;346:1221-31.

7. Salgodo Jr. W, Santos JS, Sankarankutty AK, Castro ESilva OD. Non-alcoholic fatty liver disease and obesity. Acta Cir Bras 2006;21:72-78.

8. Angulo P, Keach JC, Batts KP, Lindor KD. Independent predictor of liver fibrosis in patients with nonalcoholic steatohepatitis. Hepatology. 1999;30:1356 - 62 .

9. Wang Y, Zhou M, Lam KS, Xu A. Protective roles of adiponectin in obesity-related fatty liver diseases: mechanisms and therapeutic implications. Arq Bras Endocrinol Metabol. 2009, 53(2):201-212.

10. Luyckx FH, Lefebve PJ, Scheen AJ. Non-alcoholic steatohepatitis: association with obesity and insulin resistance, and influence of weight loss. Diabetes Metab. 2000;26:98-106.

11. Donnelly KL, Smith CI, Schwarzenberg SJ, Jessurun J, Boldt MD, Parks EJ. Sources of fatty acids stored in liver and secreted via lipoproteins in patients with non alcoholic fatty liver disease. J Clin Invest. 2005;115:1343-51.

12. Jin HB, Gu ZY, Yu CH, Li YM. Association of nonalcoholic fatty liver disease with type-II diabetes: clinical features and independent risk factors in diabetic fatty liver patients. Hepatobiliary Pancreat Dis Int. 2005;4:389-92.

13. Deng HM, Xiao CQ, Par HL. Analysis of associated factors in type-II diabetic patients with fatty liver. J Clin Intern Med. 2003;20:22-25.

14. Sharma D, Singh SK, Gupta P, Singh N et al. Role of Insulin Resistance in Non Alcoholic Fatty Liver in Obese Type-2 Diabetic Subjects. Journal of Medical Science and Clinical Research. July 2014, Volume 2 Issue 7:1838-1843.

15. Sharma D, Singh SK et al. Status of Liver Enzymes in Non Alcoholic Fatty Liver in Obese Type-2 Diabetic Subjects. International Journal of Science and Research. July 2014, Volume 3 Issue 7:1652-1653.

16. Vazarova B, Stefan N, Lindsay RS, Saremi A, Pratley $\mathrm{RE}, \mathrm{Bogardus} \mathrm{C}$ et al. High alanine aminotransferase is associated with decreased hepatic insulin sensitivity and predicts the development of type-II diabetes. Diabetes 2002;51:1889-95. 
17. Marchesini G, Brizi M, Morselli Labate AM, Bianchi G, Bugianesi G, McCullough AJ, Forlani G, Melchionda N. Association of non-alcoholic fatty liver disease to insulin resistance. Am J Med. 1999;107:450-455.

18. Duseja A, Das A, Das R, Dhiman RK, Chawla Y, Bhansali A, et al. The clinicopathological profile of Indian patients with nonalcoholic fatty liver disease (NAFLD) is different from that in the West. Dig Dis Sci. 2007;52:2368-74.

19. Kerner A, Avizohar O, Sella R, et al.: Association between elevated liver enzymes and $\mathrm{C}$-reactive protein: possible hepatic contribution to systemic inflammation in the metabolic syndrome. Arterioscler Thromb Vasc Biol. 2005;25(1):193-197.

20. Lizardi-Cervera J, Chavez-Tapia NC, Pérez-Bautista O, Ramos MH, Uribe M. Association among Creactive protein, Fatty liver disease, and cardiovascular risk. Dig Dis Sci. 2007, 52(9):2375-2379.

21. Kogiso T, Moriyoshi Y, Shimizu S, Nagahara H, Shiratori K.: High-sensitivity C-reactive protein as a serum predictor of nonalcoholic fatty liver disease based on the Akaike Information Criterion scoring system in the general Japanese population. J Gastroenterol. 2009;44(4):31321.

22. Wieckowska A, McCullough AJ, Feldstein AE: Noninvasive diagnosis and monitoring of nonalcoholic steatohepatitis: present and future. Hepatology. 2007;46(2):582-589.
23. Verrijken A, Francque S, Mertens I, et al., Visceral adipose tissue and inflammation correlate with elevated liver tests in a cohort of overweight and obese patients. Int J Obes. Feb. 2010;34:9:899-907.

24. Koruk M, Taysi S, Savas MC, et al. Serum levels of acute phase proteins in patients with nonalcoholic steatohepatitis. Turk J Gastroenterol. 2003, 14(1):12-17.

25. Yoneda M, Mawatari H, Fujita K, et al.: Highsensitivity $\mathrm{C}$-reactive protein is an independent clinical feature of nonalcoholic steatohepatitis (NASH) and also of the severity of fibrosis in NASH. $J$ Gastroenterol. 2007, 42(7):573-782.

26. Marchesini G, Moscatiello S, Di Domizio S et al. Obesity-associated liver disease. J Clin Endocrinol Metab. 2008,93:S74-80.,

27. Donnelly KL, Smith CI, Schwarzenberg SJ, Jessurun J, Boldt MD, et al. Sources of fatty acids stored in liver and secreted via lipoproteins in patients with nonalcoholic fatty liver disease. The Journal of clinical investigation. 2005, 115:1343-1351.

How to cite this article: Singh SK, Sharma D, Singh N, Kumar R, Gautam P. Status of high sensitive C- reactive protein (hs-CRP) in NAFLD obese type-2 diabetic subjects. Int $\mathrm{J}$ Clin Biochem Res. 2018;5(3):361-364. 